\title{
DIAGNOSTIC CHALLENGE OF A LOCALLY ADVANCED LESION: CASE REPORT OF PRIMARY BREAST ANGIOSARCOMA
}

Persis Araújo Oliveira', Juliana Campelo Aragão Bitencourt' ${ }^{1}$, Lorena Natali Cardoso Fernandes Caldas

${ }^{1}$ Hospital Universitário Professor Alberto Antunes - Maceió (AL), Brazil.

Introduction: Primary angiosarcoma of the breast $(\mathrm{PAOB})$ is a class of extremely rare sarcomas, with an incidence rate of $1 / 2,000$ cases of breast cancer worldwide. It is more frequent in 20- and 50-year-old women without history of previous cancer and commonly described in the left breast. Clinical presentation can be the same as usual breast cancer and histology can mimic poorly differentiated ductal carcinoma, which is why immunohistochemistry should be performed. Swelling, a feeling of fullness and exponential growth within the breast are frequent complaints, as noted by Kunkiel et al. in their series of case reports. The natural history of PAOB is only partly understood, suggesting that the lesion begins within the mammary parenchyma and then infiltrates skin and subcutaneous tissue nearby. The predominant management has been mastectomy, mainly, or sectorectomy with clear margins in cases of conservative breast surgery. Adjuvant therapies are not associated with improved survival, except for adjuvant chemotherapy in localized tumors of $5 \mathrm{~cm}$ or more. Case report: S.O.S., a 32-year-old woman, identified breast asymmetry in 2017, during the lactation period, presence of mild pain and swelling in the left breast. She was admitted to the breast cancer and benign lesions outpatient clinic at Professor Alberto Antunes University Hospital in February 2019. She held a BI-RADS 4 breast magnetic resonance imaging (MRI) in January 2019, which suggestedan irregular mass in the left breast, probably of vasculolymphatic nature; also showed core biopsy in February 2019: low-grade PAOB. In April 2019, she underwent a modified radical mastectomy of the left breast with ipsilateral lymphadenectomy. Due to the large extent of the lesion, an entire cutaneous area of left anterior hemithorax was resected, and thoracoepigastric flap was used to close the left hemithorax. An anatomopathological report diagnosed PAOB grade I. In July 2019, immunohistochemistry corroborated the diagnosis of PAOB with CD31 positive; positive von Willebrand factor (Factor VIII - polyclonal Rabbit) and ki67 positive for $25 \%$ of neoplastic cells. In the fourth month after the surgery, the patient started adjuvant radiotherapy, concluding it in October 2019. In post-treatment follow-up, in January 2021, she was referred to the breast reconstruction program, awaiting the procedure until this report was made. 